\title{
SIMULACIÓN Y OPTIMIZACIÓN DE SISTEMA DE MANUFACTURA CON CRITERIOS ECONÓMICO Y AMBIENTAL
}

\author{
SIMULATION OPTIMIZATION OF A MANUFACTURING SYSTEM WITH \\ ECONOMIC AND ENVIRONMENTAL CRITERIA
}

\author{
Héctor Darío Enriquez ${ }^{1 *}$, Sebastián Federico Kolodziej²
}

\begin{abstract}
RESUMEN
En la simulación y optimización de un sistema de manufactura se buscan los parámetros de entrada para obtener el mejor desempeño del sistema, considerando en muchos casos un único objetivo o medida de desempeño. Sin embargo, muchas decisiones implican múltiples criterios, usualmente en conflicto. Actualmente, las empresas se ven exigidas a mejorar su rentabilidad, como también presionadas a reducir sus impactos en el ambiente y utilizar racionalmente la energía. En este trabajo se estudió el proceso de manufactura de artículos de madera de una pequeña empresa, para determinar alternativas de rediseño, con criterios económico y ambiental en simultáneo. Se construyó un modelo de simulación del sistema, el cual fue validado y verificado, y un modelo de optimización para el mismo, en el que se combinaron dos objetivos en una única función, incorporándose en la misma las preferencias de los decisores respecto a cada criterio. Los resultados, combinando ambos criterios en simultáneo, muestran substanciales mejoras en el beneficio, generando menos emisiones que si se persiguiese solamente el objetivo económico. Se concluye que la simulación en conjunto con la optimización multiobjetivo puede mejorar el desempeño económico-ambiental de los sistemas fabriles del sector maderero en forma apreciable, incluso en pequeños establecimientos.
\end{abstract}

Palabras clave: Simulación, Optimización, Metaheurística, Toma de Decisiones Multicriterio, Impacto ambiental, Industria maderera.

\section{ABSTRACT}

With the simulation and optimization of a manufacturing system, the input parameters are sought to obtain the best performance of the system, considering in many cases a single objective or measure of performance. However, many decisions involve multiple criteria, usually in conflict. Currently, companies are required to improve their profitability as well as

\footnotetext{
${ }^{1}$ Departamento de Ingeniería Industrial, Facultad de Ingeniería, Universidad Nacional de Misiones (FI-UNaM), Oberá, Argentina. enriquez@fio.unam.edu.ar. orcid.org/0000-0003-0475-5008.

${ }^{2}$ Departamento de Ingeniería Industrial, Facultad de Ingeniería, Universidad Nacional de Misiones (FI-UNaM), Oberá, Argentina. orcid.org/0000-0002-2532-180X
} 
to reduce their impact on the environment and use energy rationally. In this paper, the wood products manufacturing process of a small company was studied to determine redesign alternatives, with simultaneous economic and environmental criteria. A simulation model of the system was built, which was validated and verified, and an optimization model for it in which two objectives were combined in a single function, incorporating in it the preferences of the decision makers with respect to each criterion. Results expose that combining both criteria it is possible to achieve substantial improvements in the benefit, at the same time that generating less emissions than if only the economic objective were pursued. It is concluded that simulation and optimization combining both criteria can improve economic-environmental performance in wood manufacturing systems, even in small companies.

Keywords: Simulation, Optimization, Metaheuristics, Multicriteria Decision Making, Environmental impact, Wood industry.

\section{INTRODUCCIÓN}

Los modelos se han convertido en herramientas imprescindibles en casi cualquier proceso de toma de decisiones relativas a sistemas complejos, como es el caso de los sistemas de manufactura. Dicho término se refiere a un conjunto de procesos en los cuales se combinan máquinas, herramientas, energía, información y trabajo humano, siguiendo una secuencia de operaciones para transformar materiales en productos de mayor valor, para ser comercializados posteriormente (Esmaeilian et al., 2016; Mourtzis et al., 2014).

Un sistema de manufactura puede estudiarse mediante la experimentación directa o utilizando un modelo. A menudo se recurre a la segunda opción, por razones de costo, tiempo, riesgo, ética, o simplemente porque la experimentación real no es posible (Law, 2008, 2015a; Ríos et al., 2009). A su vez, se encuentran distintos tipos de modelos: a) modelos físicos; b) modelos simbólicos, los cuales se subdividen en modelos matemáticos y no matemáticos; y c) modelos mentales (Ríos et al., 2009). Para analizar sistemas de manufactura y evaluar su desempeño, en particular es frecuente recurrir a modelos matemáticos analíticos o de simulación (Elizandro \& Taha, 2012; Law 2015a). Si bien muchos modelos analíticos pueden ser útiles, numerosos sistemas son estocásticos y muy complejos, siendo en tales casos la simulación la única opción viable para evaluar su desempeño (Elizandro \& Taha, 2012; Hillier \& Lieberman, 2015; Law 2015a; van Dijk et al., 2015; Yegul et al., 2017). Además, los avances en la informática, nuevos paradigmas de modelado y software especializado, permiten en muchos casos resultados prácticamente tan precisos como con los métodos analíticos (Chica et al., 2017).

La simulación consiste en crear un modelo matemático en un programa de computadora que describa el comportamiento del sistema de interés, proceder a evaluar numéricamente el modelo y realizar experimentos para obtener datos que permitan estimar el comportamiento del sistema real (Hillier \& Lieberman, 2015; Law, 2015a; Mourtzis et al., 2014; Ríos et al., 2009). Los modelos de simulación pueden clasificarse de diversas formas, como presentan Kleijnen (2015), Mourtzis et al. (2014) y Law (2015a). Para el diseño y análisis de sistemas de manufactura, la simulación por eventos discretos es una de las técnicas más utilizadas (Elizandro \& Taha, 2012; Guasch et al., 2003; Law 2015a; Negahban \& Smith, 2014; Sproedt et al., 2015; Yegul et al., 2017). En particular, en procesos de aprovechamiento de madera, tanto en aserrío como en productos manufacturados, se vienen utilizando modelos de simulación por computadora desde hace medio siglo, como ejemplos se encuentran Aune (1974) y Reynolds y Gatchell (1969), otros son Anderson (1983), Kline et al. (1992), Baesler 
et al. (2002), y Wery et al. (2015).

Tradicionalmente, recursos como los materiales, humanos y monetarios son los que se preponderan en la planificación y control de sistemas de manufactura. A pesar de ello, actualmente las empresas se ven exigidas a incorporar en los análisis métricas de sostenibilidad (Pawlewski \& Borucki, 2011; Tsai et al., 2015; Thiede et al., 2013). Las manufactureras se encuentran entre las principales responsables de la contaminación del mundo, por lo que están sujetas a presiones para reducir sus impactos, mientras deben continuar suministrando productos y servicios de alto valor para el cliente, a precios competitivos (Garwood et al., 2018; Sproedt et al., 2015). Entre las presiones se encuentra el uso racional de la energía, cada vez más importante por el impacto ambiental, pero también por el aumento de precios de los recursos (Herrmann et al., 2011; Sproedt et al., 2015).

Modelar el consumo de energía puede ayudar a comprender mejor cómo y dónde se utilizan los recursos, incorporando elementos adicionales para la optimización de los sistemas de producción (Johansson \& Thollander, 2018; Pawlewski \& Borucki, 2011; Thiede et al., 2013); como también evitar multas por la contaminación (Wróbel \& Oleśków, 2014).

Existe entonces la necesidad de incluir en el diseño, evaluación y control de sistemas de manufactura, criterios de sostenibilidad. Sin embargo, a los decisores de estos sistemas no siempre les resulta sencillo traducir estos criterios en metas, evaluar costos y beneficios, y poner en práctica las medidas de mejoramiento necesarias a nivel de planta (Sproedt et al., 2015). La simulación ofrece oportunidades para resolver estos problemas. No obstante, según Sproedt et al. (2015) la evidencia disponible en la literatura indica que la toma de decisiones con criterios de sostenibilidad o de "ecoeficiencia" en sistemas de producción es aún un proceso inmaduro. Los mismos autores señalan además las siguientes barreras para implementar medidas que mejoren el desempeño ambiental en la producción: limitado conocimiento de los decisores respecto a este tipo de mejoras; limitaciones en cuanto a los recursos para realizar estos análisis; dificultades para obtener información sobre el impacto ambiental, y equilibrar los costos con los beneficios ambientales.

Sproedt et al. (2015) también mencionan que con simulación por eventos discretos los decisores pueden obtener información sobre las interrelaciones complejas entre los desempeños económico y ambiental. Sin embargo, la integración de aspectos ambientales en la simulación de sistemas de manufactura aún no se ha convertido en una práctica consolidada (Sproedt et al., 2015).

Algunos programas de simulación comercial, incorporan funciones para análisis ambientales, que incluyen la generación de emisiones, residuos y uso de energía. No obstante, en general dichas capacidades continúan siendo limitadas en la actualidad (Garwood et al., 2018; Herrmann et al., 2011; Pawlewski \& Borucki, 2011; Thiede et al., 2013; Sproedt et al., 2015).Este enfoque es relativamente reciente, observándose numerosas publicaciones durante la última década. Johansson y Thollander (2018) señalan a Solding (2008) como uno de los primeros en utilizar simulación por eventos discretos para el uso eficiente de la energía en plantas de manufactura. A continuación se describen otros ejemplos. Heilala et al. (2008) presentan el proyecto de una herramienta de simulación que combina el análisis de la eficiencia de la producción con el impacto ambiental, utilizando como métricas el consumo de energía y generación de contaminantes. Herrmann et al. (2011) presentan un procedimiento para simular procesos de manufactura, con orientación al uso de la energía y lo aplican en industrias de fundición de aluminio y fábrica de tejidos. Diaz et al. (2013) desarrollan una metodología de simulación que integra estrategias lean y green para sistemas de manufactura, presentando un caso en una industria automotriz. Wróbel y Oleśków (2014) diseñan el proceso de una pequeña industria de artículos de madera utilizando simulación, considerando requisitos de seguridad y protección ambiental. Sproedt et al. (2015) proponen una metodología de simulación que integra el análisis de ciclo de vida para evaluar el desempeño ambiental de procesos de manufactura. Garwood et al. (2018), Pawlewski \& 
Borucki (2011) y Thiede et al. (2013) realizan revisiones de la literatura sobre herramientas de simulación y sus capacidades para modelar aspectos ambientales y uso de energía.

A diferencia de otros métodos analíticos, la simulación por sí misma no conduce a una solución óptima (van Dijk et al., 2015); no implica llegar a una solución resolviendo el modelo, sino que a través de la experimentación con distintos parámetros de entrada (inputs) puede observarse que sucede con la medida de desempeño del modelo (output). Es decir, que el objetivo principal de la experimentación con un modelo de simulación es encontrar la combinación de inputs que permitan obtener el mejor output del sistema. El problema en cuestión puede representarse entonces mediante la expresión (1).

$$
\text { Minimizar (o Maximizar) } \mathrm{g}(\mathrm{x}), \mathrm{x} \in \Theta
$$

Donde $x=\left(x_{1}, x_{2}, \ldots, x_{m}\right)$ es el vector de los $m$ - inputs y $\Theta$ es la región factible dada por un conjunto de restricciones. La función objetivo $\mathrm{g}(\mathrm{x})=\mathrm{E}[\mathrm{Y}(\mathrm{x}, \varepsilon)]$ da como resultado un escalar, siendo el valor esperado de la variable aleatoria $Y(X, \varepsilon)$ representativa de la medida de desempeño del sistema, con $\varepsilon$ designando la fuente de aleatoriedad (Andradóttir, 2015; Choi \& Kang, 2013; Figueira \& Almada, 2014; Fu, 2002; Fu et al., 2005; Hong et al., 2015, Nelson, 2013). Los parámetros controlables x reciben normalmente el nombre de variables de decisión, mientras que $\mathrm{g}(\mathrm{x})$ es denominada función objetivo del problema.

Una manera de analizar el comportamiento de las medidas de desempeño de un modelo es ver a la simulación como la propia función $\mathrm{g}(\mathrm{x})$. En ciertos casos puede aproximarse la función $\mathrm{g}(\mathrm{x})$ en términos algebraicos, o bien el modelo puede considerarse como una "caja negra" que realiza la conversión input/output (Amaran et al., 2016; Better et al., 2015; Laguna \& Marklund, 2013).

Se distinguen dos tipos de problemas de optimización en simulación, según el conjunto de alternativas sea finito (discreto) o continuo. Para cada tipo de problema, existen métodos analíticos como los que describen Fu (2015), Hunter et al. (2017) y Ríos et al. (2009). Relativamente son pocos los algoritmos que abordan simultáneamente variables continuas y discretas (Amaran et al., 2016). Nelson (2013) también distingue dos tipos de problemas, pero según el conjunto de restricciones sea determinístico o estocástico. Esto viene dado por la posibilidad (o no) de determinar con certeza si cada escenario (x) a simular es factible o infactible. Las restricciones estocásticas presentan mayores dificultades para la optimización.

Muchos programas informáticos para simulación incorporan algoritmos de optimización metaheurísticos (Hong et al., 2015; Laguna \& Marklund, 2013; Law, 2015a). Estos métodos, si bien proveen buenas soluciones, no garantizan que se alcance el óptimo. Por lo tanto, de aquí en adelante el término "optimización" se refiere a un proceso de "búsqueda del óptimo" por medio de un programa informático.

La optimización generalmente se realiza en forma iterativa (figura 1): a) realizar corridas del modelo; b) analizar el output; c) evaluar las medidas de desempeño (KPI- Key Performance Indicators); d) seleccionar nuevos valores para los parámetros y ajustar el input; e) realizar otras corridas del simulador. Este ciclo se repite un número suficiente de veces, de acuerdo a una planificación o bien alguna regla de detención establecida (Better et al., 2015; Choi \& Kang, 2013, Fu, 2002, Law, 2015a). Por la naturaleza de las metaheurísticas, suele ser difícil determinar la regla de detención (Fu, 2002, 2015). Tres posibles reglas son (Hillier \& Lieberman, 2015): 1) fijar el número de iteraciones; 2) fijar el tiempo de procesamiento computacional; 3 ) detener tras un número fijo de iteraciones sin mejora alguna. La rutina de optimización puede implementarse tanto en forma interna como externa al modelo de simulación (Chica et al., 2017; Kibira \& Shao, 2017). 


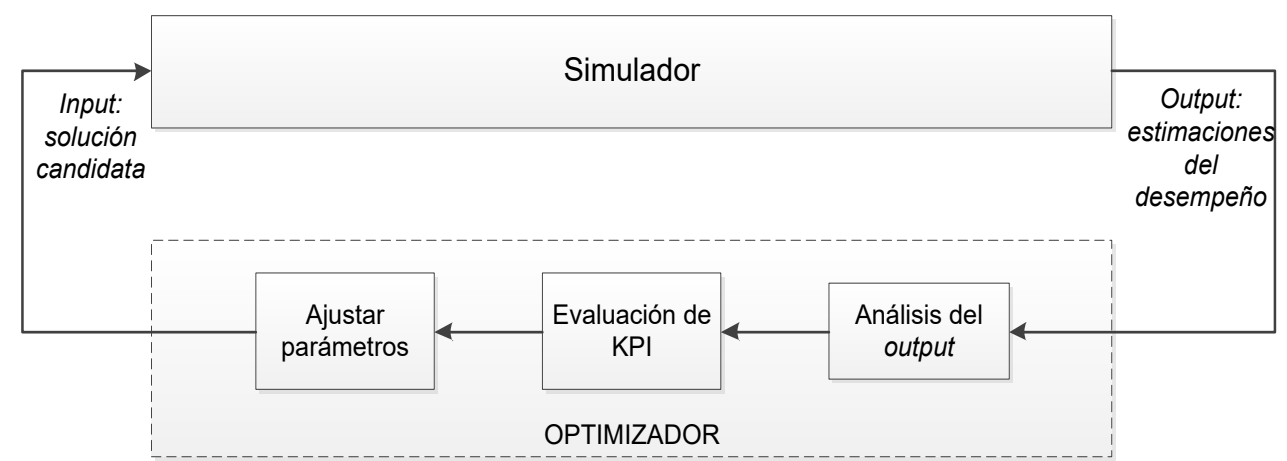

Figura 1. Funcionamiento de un optimizador para simulación.

Fuente: Better et al. (2015); Choi y Kang (2013); Fu (2002).

Figueira y Almada (2014) presentan una clasificación de los métodos de optimización para simulación. Pueden encontrarse detalles y aplicaciones en distintos software en Amaran et al. (2016), Fu (2002, 2015) y Law (2015a).

En general, en el estudio de los sistemas reales existen múltiples parámetros de desempeño que se desean optimizar, por lo tanto pueden establecerse múltiples funciones objetivo, en lugar de una como se presentó en la expresión (1). Usualmente dichas funciones objetivo se encuentran en conflicto. En este tipo de problemas no se halla una solución óptima para todos los objetivos en simultáneo, sino que la solución es de compromiso, dentro de un conjunto de soluciones denominadas Pareto-óptimas o no-dominadas (Andradóttir, 2015; Elarbi et al., 2017; Hunter et al., 2017). Las preferencias subjetivas del decisor son la base para seleccionar una solución dentro de un conjunto optimal de Pareto (Fontes \& Gaspar, 2010).

Los métodos de optimización multiobjetivo se pueden clasificar en dos tipos (Deb, 2014; Fontes \& Gaspar, 2010): los que se basan en optimizar una sola función, que se denominan métodos basados en preferencias de los decisores, los cuales posibilitan obtener una solución de compromiso particular; y los que tratan de identificar el conjunto optimal de Pareto, y a partir de este decidir una solución. Entre los primeros, los más conocidos combinan las distintas funciones objetivo en una única función. Una manera sencilla de hacerlo es como una suma ponderada de funciones, asignando pesos apropiados a cada una (Deb, 2014; Elarbi et al., 2017; Fontes \& Gaspar, 2010; Fu, 2002). Otro procedimiento es optimizar un objetivo a la vez, mientras los otros se tratan como restricciones del modelo (Andradóttir, 2015, Deb, 2014, Elarbi et al., 2017). Banks et al. (2005) también consideran este último método, y agregan los siguientes: 1) combinar todas las medidas de desempeño en una sola representativa, que luego es optimizada; 2) optimizar una medida de desempeño a la vez, y después evaluarla con respecto al cumplimiento de los demás objetivos. Baesler y Sepúlveda (2000) presentan el uso de programación por metas para modelos de simulación. Los distintos autores destacan para los métodos basados en las preferencias de los decisores el inconveniente de asignar ponderaciones adecuadas y la sensibilidad a dichos pesos. Respecto a los métodos que tratan de encontrar en el conjunto optimal de Pareto, o como los denomina Deb (2014) métodos "ideales", Fontes \& Gaspar (2010) enfatizan que los mismos pueden resultar imprácticos en muchos problemas reales, en términos computacionales, y también que la comprobación de la optimalidad puede ser inviable, por lo que sugieren que se evalúe un conjunto finito de soluciones. Deb (2014) en tanto, pone de manifiesto que, si bien los métodos ideales son más objetivos, si las preferencias relativas están disponibles no hay razón para buscar otras soluciones de compromiso, y podría aplicarse el método basado en preferencias.

Existe software para optimizar modelos de simulación con objetivos múltiples (Fu et al., 2005; 
Laguna, 2011). No obstante y a pesar de los avances teóricos y prácticos, los problemas multiobjetivo en simulación aún presentan desafíos para la investigación (Fontes \& Gaspar, 2010; Fu et al., 2014; Lin et al., 2013).

En este trabajo se analiza el proceso de manufactura de artículos de madera de una pequeña empresa, localizada en la ciudad de Oberá, provincia de Misiones, República Argentina. Los decisores en esta empresa deseaban mejorar el beneficio económico de la producción, pero a su vez generando menor impacto ambiental. Por las mencionadas oportunidades que ofrece la simulación para incorporar al análisis criterios ambientales, además de sus ventajas para estudiar sistemas de manufactura, se aplica esta técnica para abordar el sistema en cuestión. Al momento de redactar este artículo, no se tiene conocimiento de otras aplicaciones de simulación en plantas del sector maderero que busquen la optimización con criterios económico y ambiental simultáneamente.

Considerando la prioridad de los decisores hacia el beneficio económico, se formula la siguiente pregunta de investigación ¿es posible determinar una configuración para el sistema que genere un incremento significativo en el beneficio, pero con menor impacto ambiental que si se persiguiese únicamente el objetivo económico? Luego se definen los siguientes objetivos: desarrollar un modelo de simulación del sistema, que permita la estimación del beneficio e impacto sobre el medioambiente; después de validado y verificado el modelo, elaborar un modelo de optimización para éste, que incluya una función económica y una ambiental; y comparar los resultados contra configuraciones que optimicen, en forma separada, los criterios económico y ambiental.

\section{METODOLOGÍA}

Para llevar a cabo el proyecto de simulación se siguió un proceso compuesto por tres macrofases (Chwif et al., 2013): conceptualización; programación y análisis. Cada una de estas fases incluyó un conjunto de pasos, que se concretaron siguiendo el proceso propuesto por Law (2015a). A continuación se sintetiza dicho proceso.

- Formulación del problema: El caso estudiado corresponde al proceso de fabricación de artículos de madera en una pequeña empresa localizada en Oberá, Argentina. Se elaboran tres versiones del artículo, diferentes y en dos calidades. En el sector de producción hay cinco operarios, un autoelevador, una línea de producción, y el proceso es por lotes. El proceso completo se resume a continuación:

- Como materia prima principal se tienen varillas de madera dura, que ingresan en paquetes compactos, luego son inspeccionadas, se clasifican y se acomodan al aire libre para su secado natural.

- La línea de producción está constituida por las siguientes actividades de manera secuencial: primer torneado; despuntado (corte en las puntas); segundo torneado en uno de los extremos; lijado; roscado en el otro extremo (si correspondiese).

- Posteriormente los artículos terminados pasan por una selección, donde se clasifican según su calidad, luego son empaquetados, cada paquete es palletizado y finalmente se almacena antes de su expedición.

- Los residuos y desperdicios de madera (aserrín, trozos de madera, listones defectuosos, etc.) se comercializan a otras empresas para ser utilizados como combustible (mediante la quema). 
Fueron realizadas reuniones entre los analistas y decisores (responsables de la empresa), se enunciaron objetivos del proyecto y preguntas específicas que debían responderse, alcance del modelo, los KPI a utilizarse para evaluar las propuestas, tiempo para realizar el proyecto, entre otras cuestiones. Interesaban a los decisores alternativas de diseño para incrementar el beneficio económico de la producción, causando el menor impacto ambiental. Las alternativas de diseño quedaron definidas por las siguientes modificaciones posibles: variar la frecuencia entre pedidos de materia prima y el tamaño de los lotes; incorporar un horno para acelerar el secado de materia prima (conservando en paralelo el secado natural), que utilice como combustible parte o la totalidad de los residuos de madera; agregar un torno (para el primer torneado) junto con un operario para su utilización; modificar el tamaño de los lotes de producción.

Se establecieron dos objetivos: obtener el mayor beneficio económico, con el menor impacto ambiental. Los decisores expresaron mayor importancia por el beneficio económico. Para el primer objetivo se midió la diferencia entre ingresos y costos (en \$ semanales), mientras que para el segundo se evaluó la generación de gases de efecto invernadero $(\mathrm{GEI})$ en kilogramos de $\mathrm{CO}_{2}$ equivalentes $\left(\mathrm{CO}_{2 \mathrm{eq}}\right)$ semanales, a causa de la utilización de energía eléctrica y térmica.

Recolección de datos y definición del modelo conceptual: Para el modelo conceptual se especificaron supuestos y consideraciones, y se elaboraron distintos diagramas. Como principales supuestos y consideraciones, se asumió que los operarios están especializados en una o más actividades establecidas, por lo que no pueden cambiar de puestos de trabajo; la materia prima es siempre de la misma calidad, por lo que la proporción de fallas es constante; las paradas en cada máquina por fallas, cambio de herramientas $u$ otras tareas de mantenimiento se descontaron del tiempo disponible de la máquina, modelando mediante un factor de eficiencia; de manera similar para los recursos humanos del proceso, para modelar ausencias o interrupciones, se adoptaron factores de disponibilidad; los tiempos para tareas generales en el establecimiento, como limpieza y orden, no fueron tenidos en cuenta.

Se utilizaron distribuciones de probabilidad para parametrizar el proceso. También se relevaron los costos de las actividades, de los recursos humanos, materia prima, gestión del inventario, amortizaciones y costos de operación para el torno adicional y el horno de secado, en caso de ser incorporados al proceso, ingresos por ventas y por recuperación de residuos y desperdicios. No se consideraron multas a causa de la contaminación.

Las emisiones de GEI fueron cuantificadas considerando las emisiones directas (provenientes de fuentes que pertenecen o son controladas por la empresa) e indirectas (que provienen de la generación de electricidad, calor, vapor, etc., de origen externo, consumidos por la organización) (Franchetti \& Apul, 2013). Las primeras fuentes provienen del consumo de combustible fósil por el autoelevador y la quema de madera para el funcionamiento del horno de secado; para las segundas se consideraron las emisiones indirectas a causa de la generación de energía eléctrica, para abastecer el consumo de los equipos que se utilizan en el proceso. Las emisiones de GEI fueron calculadas con la expresión (2).

\section{Emisiones de GEI ( $\mathrm{t}$ GEI )= Dato de actividad $x$ Factor de emisión}

El dato de actividad se obtuvo, para el caso de combustiones por ejemplo para el autoelevador, mediante el producto del consumo de combustible (en masa o volumen) y el poder calorífico inferior (PCI) del combustible, en dicho caso gasoil. Para el uso de energía eléctrica, el dato de actividad correspondió al consumo de la instalación (expresado en $\mathrm{kWh}$ ), que se obtuvo mediante la potencia de cada máquina eléctrica y el periodo de funcionamiento. El factor de emisión depende del tipo y características del proceso de transformación química y tipo de combustible. En el caso del combustible fósil, el dato se 
obtuvo de Tablas (Unzalu, 2012). Para el factor de emisión eléctrico, se utilizaron datos brindados por el Ministerio de Energía y Minería de la República Argentina (2016).

Construcción del modelo computacional: se utilizó el software de simulación SIMUL8.

Este software permitió la construcción de un modelo dinámico con características visuales del sistema, de manera sencilla, directamente incorporando objetos para representar las partes del mismo y sus interacciones (Elder, 2014; Omogbai \& Salonitis, 2016; SIMUL8 Corporation, 2018). Proporcionó también una variedad de indicadores de desempeño del proceso, costos, ingresos y beneficios económicos, e integró el análisis de emisiones de GEI. También facilitó las funciones necesarias para la experimentación y análisis de resultados. En la figura 2 se muestran capturas de pantalla del modelo de simulación en SIMUL8.
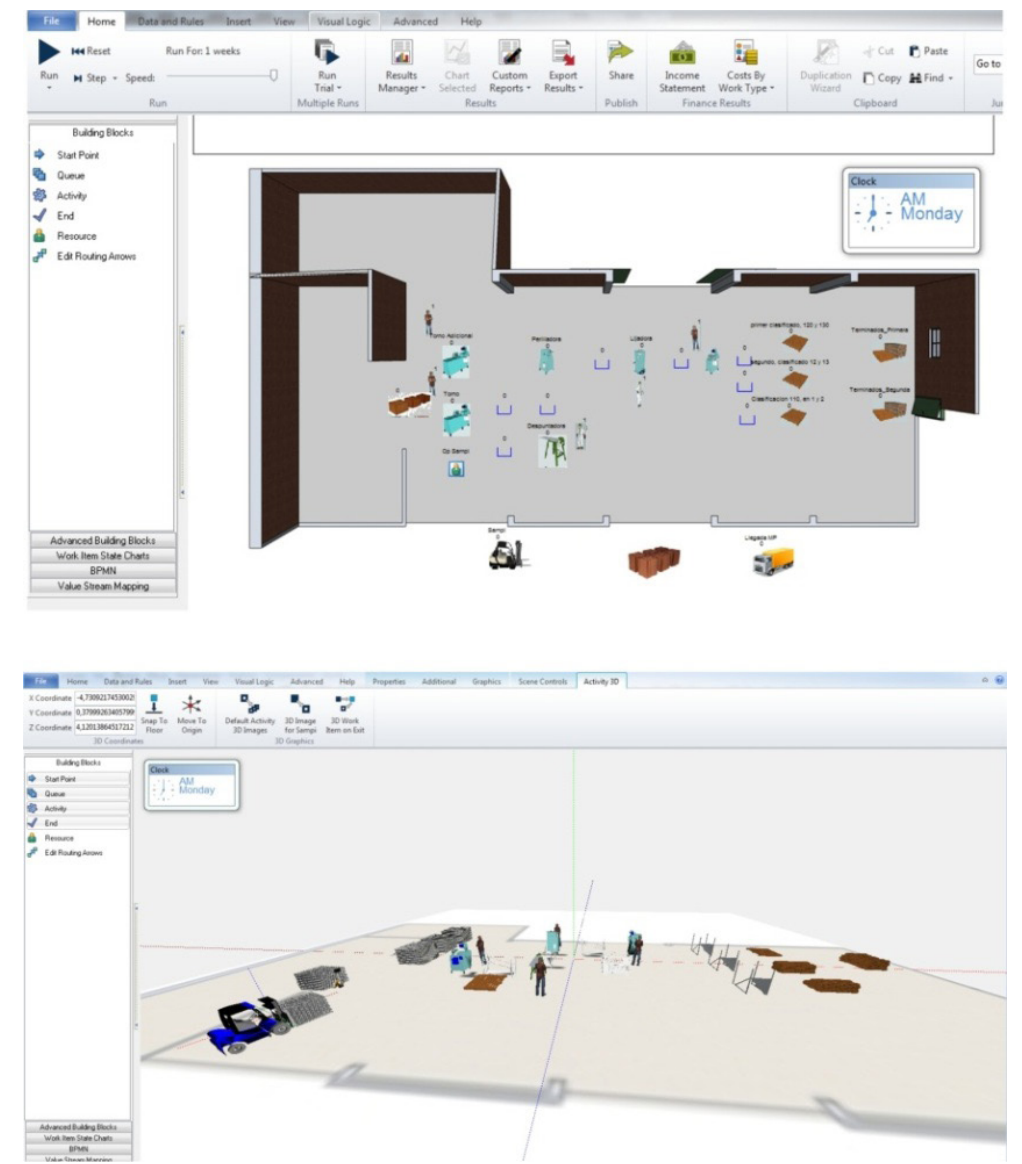

Figura 2. Modelo de simulación desarrollado.

- Validación y verificación: se efectuaron validaciones de los datos, de los modelos conceptual y operacional, y fue verificado el modelo computacional (Guasch et al., 2003; Law, 2015a; Sargent, 2015). Se analizaron los supuestos del modelo conceptual entre analistas, responsables y personal experto de la empresa. Al tratarse de un sistema existente, pudo validarse el output del modelo computacional, con datos reales. También se efectuaron validaciones superficiales o informales, al consultarse directamente al personal experto respecto al comportamiento del modelo y sus resultados.

- Diseño, ejecuciónyanálisis de experimentos: previoalaoptimización, setomaron decisiones 
de la longitud de cada simulación, número de réplicas independientes y periodo de calentamiento(warmup), pararealizarsimulacionesenestadoestacionario.Paradeterminar el warm up se aplicó el método de Welch (Law 2015a, 2015b), obteniéndose una duración de 4 semanas. El número de réplicas necesarias fue de 32, obtenido con la función trial calculatordelsoftwareSIMUL8(Elder,2014). Lalongituddecadasimulaciónfuede1 semana.

\section{Optimización multiobjetivo del modelo de simulación}

Laoptimización del modelo de simulación fue realizada con el software OptQuest(OptTek, 2018), integrado en SIMUL8. Los métodos de optimización de este software son las metaheurísticas búsqueda tabú, búsqueda esparcida y redes neuronales artificiales (Amaran et al., 2016; Better et al., 2015; Fu, 2002; Fu et al., 2005; Laguna, 2011; Law, 2015a; Yegul et al., 2017).

Las variables de decisión o inputs para la optimización se definieron así:

$\mathrm{x} 1$ : frecuencia entre pedidos de materia prima [días].

x2: tamaño de los lotes de materia prima [miles de unidades].

$\mathrm{x} 3$ : incorporar $(=1)$ o no $(=0)$ un horno para el secado de la materia prima.

$x 4$ : tamaño de lote de producción [unidades].

$x 5$ : incorporar $(=1)$ o no $(=0)$ un torno y un operario.

Los dos objetivos del problema se precisaron con las funciones (3) y (4).

$$
\begin{aligned}
& \text { Maximizar } g(x)=g\left(x_{1}, x_{2}, x_{3}, x_{4}, x_{5}\right) \quad[\text { Beneficio, en } \$ / \text { semana] } \\
& \text { Minimizar } h(x)=h\left(x_{1}, x_{2}, x_{3}, x_{4}, x_{5}\right) \quad\left[\text { Emisiones } C_{2}\right. \text { eq, en kg/semana] }
\end{aligned}
$$

Las funciones (3) y (4) fueron combinadas como suma ponderada en una única función a ser optimizada. Para hacer la combinación, las funciones fueron normalizadas (Baesler \& Sepúlveda, 2000). Existen diversos métodos de normalización. Mohamad y Usman (2013) afirman que no existe una regla universal para normalizar datos. Además, para ciertos casos pueden encontrase ventajas e inconvenientes entrelos métodos (García etal.,2015; Mohamad \& Usman, 2013; Patel \& Thakral, 2016). Baesler y Sepúlveda (2000) proponen dividir las funciones objetivo por los valores establecidos como metas. Larose y Larose (2015) como también García et al. (2015) presentan tres métodos: min-max; variable estandarizada Z; y escalado decimal. Este último se destaca por su sencillez (García et al., 2015) y se seleccionó por tal motivo. Las funciones normalizadas $g^{*}(x) y^{*}(x)$ que se utilizaron se presentan en (5) y (6).

$$
\begin{aligned}
& g *(x)=\frac{g(x)}{10^{d_{1}}} \\
& h^{*}(x)=\frac{h(x)}{10^{d_{2}}}
\end{aligned}
$$

En (5) y (6) d1 y d2 son los enteros más pequeños tal que máx $\left[\mid g^{*}(x\right.$ ) $\mid]<1$ y $\operatorname{máx}\left[\left|\mathrm{h}^{*}(\mathrm{x})\right|\right]<1$, respectivamente. En el caso analizado $\mathrm{d} 1=5$ y $d 2=3$. La función objetivo resultante se define por la ecuación (7).

$$
\text { Maximizar } f^{\star}(x)=f^{*}\left(x_{1}, x_{2}, x_{3}, x_{4}, x_{5}\right)=w_{1} g^{*}(x)-w_{2} h^{*}(x)
$$

El método que se utilizó es basado en las preferencias de los decisores, con las funciones agregadas como suma ponderada. Se aplicó este método ya que a priori se disponía la preferencia relativa de los decisores por los criterios y se consideró la obtención de 
una solución de compromiso particular. En (7) $w_{1}$ y $w_{2}$ son los pesos que representan las preferencias por los objetivos económico y ambiental respectivamente. En este caso, se otorgó mayor preferencia al primero, y los pesos fueron $w_{1}=0,70$ y $w_{2}=0,30$.

Conocidos los límites (restricciones) de las variables de decisión, definidos por los decisores opor condiciones propias del sistema, el modelo completo se presenta en las expresiones (8) a (12).

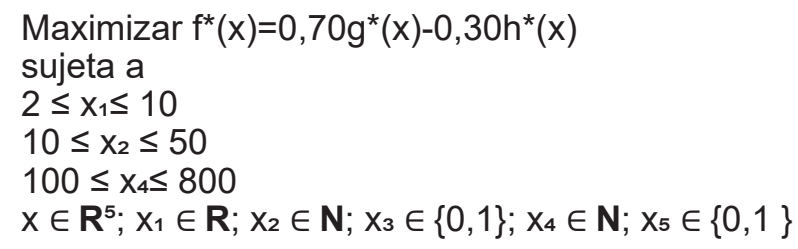

La evaluación de $\mathrm{f}^{\star}(\mathrm{x})$ se realizó mediante la ejecución de réplicas independientes del modelo de simulación. Se trata de un modelo mixto (continuo-discreto), con restricciones determinísticas.

Además de optimizarse la $f^{*}(x)$, lo cual es el objetivo principal de este trabajo, también se optimizaron por separado las funciones $g^{*}(x)$ y $h^{*}(x)$, sujetas cada una también a las restricciones (9) a (12), para realizar posteriormente comparaciones de resultados. Considerando el tiempo computacional para la ejecución de cada replicación y observando el tiempo sin mejora en la optimización de cada función, se decidió como regla de detención para el optimizador fijar el número de iteraciones en 50.

Con el conocimiento de los óptimos de cada función y del comportamiento del modelo del sistema inicial, se realizaron comparaciones entre escenarios, mediante intervalos de confianza (IC) $t$-pareados de diferencias de medias $(\mu)$ de los indicadores de desempeño. Se contrastó el modelo multiobjetivo (escenario 1: máx. $f^{*}(x)$ ) contra los demás escenarios (0: inicial; 2: máx. $\mathrm{g}^{*}(\mathrm{x})$; 3 : $\min . \mathrm{h}^{*}(\mathrm{x})$ ); y también se compararon todos los escenarios entre sí por pares. Aplicando la desigualdad de Bonferroni (Banks et al., 2005; Kleijnen, 2015; Law, 2015a), en el primer contraste cada intervalo fue evaluado a nivel $\alpha^{\prime}=\alpha / k-$ $1=0,016$, mientras que en el segundo contraste fue a nivel $\left.\alpha^{\prime}=\alpha \backslash k(k-1) / 2\right]=0,008$, con $\mathrm{k}=4$ escenarios, para obtener en cada contraste un nivel de confianza no menor a $95 \%$.

\section{RESULTADOS Y DISCUSION}

EnlaTabla1, sepresentanlasconfiguracioneseindicadoresdedesempeñoeconómicoyambiental del modelo inicial, y de los óptimos obtenidos con OptQuest para las funciones $f^{*}(x), g^{*}(x)$ y $h^{*}(x)$. En la figura 3, se representa el comportamiento de las funciones durante su optimización, cada una en forma independiente. Se observan comportamientos aproximadamente asintóticos, creciente en el caso de $g^{*}(x)$, decreciente para $h^{*}(x)$ y creciente para la función $f^{*}(x)$, resultado de haber dado mayor peso a la primera función en la combinación. Cabe recordar que con las metaheurísticas no se garantiza el alcance del óptimo global de cada función. Este resultado general es sensible a las condiciones de experimentación, especificadas en el optimizador. 


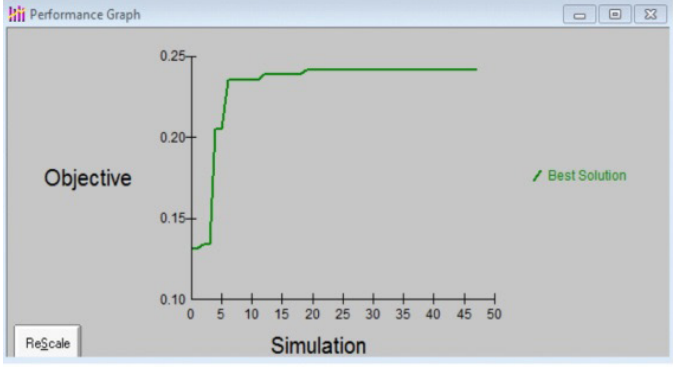

[a]

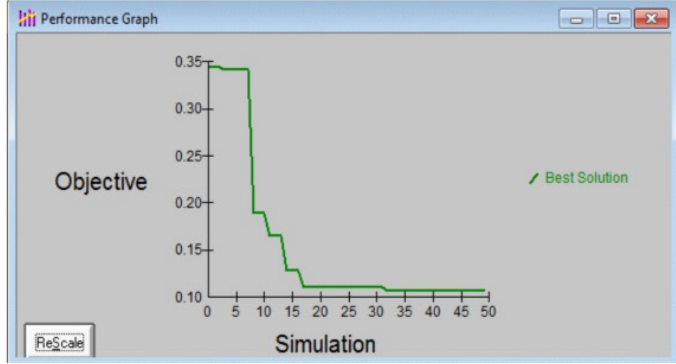

[b]

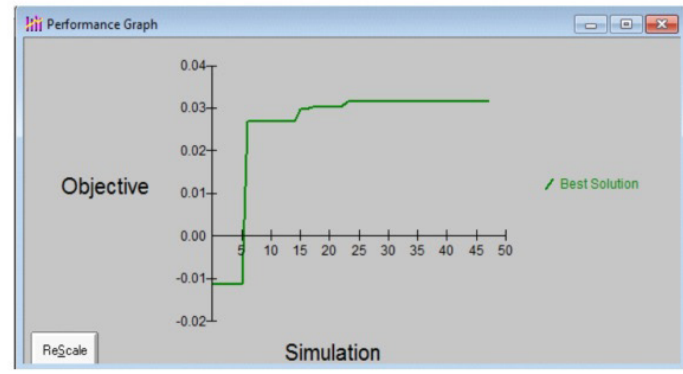

[c]

Figura 3. Gráficos de desempeño durante la optimización de las funciones [a] $g^{*}(x),[b]$ $h^{*}(x), y[c] f^{*}(x)$. con OptQuest y SIMUL8.

Tabla 1. Configuraciones e indicadores de desempeño económico y ambiental de los escenarios

\begin{tabular}{|l|c|c|c|c|c|c|c|c|c|}
\hline & \multicolumn{4}{|c|}{ Variables de decisión } & \multicolumn{3}{c|}{ Beneficio* } & \multicolumn{2}{|c|}{ Emisiones de $\mathrm{CO}_{2 \mathrm{eq}}{ }^{*}$} \\
\hline Escenarios (i) & $\mathrm{x}_{1}$ & $\mathrm{x}_{2}$ & $\mathrm{x}_{3}$ & $\mathrm{x}_{4}$ & $\mathrm{x}_{5}$ & $\begin{array}{c}\text { media } \\
\left(\mu_{\mathrm{i}}\right)\end{array}$ & $\begin{array}{c}\text { Intervalo } \\
(\alpha=0,05)\end{array}$ & $\begin{array}{c}\text { media } \\
\left(\mu_{\mathrm{i}}\right)\end{array}$ & $\begin{array}{c}\text { Intervalo } \\
(\alpha=0,05)\end{array}$ \\
\hline 0 [inicial] & 7,50 & 30 & 0 & 500 & 0 & 0,157 & $(0,146 ; 0,169)$ & 0,340 & $(0,337 ; 0,344)$ \\
\hline 1 [máx $\left.\mathrm{f}^{*}(\mathrm{x})\right]$ & 2,53 & 43 & 0 & 800 & 1 & 0,232 & $(0,228 ; 0,235)$ & 0,458 & $(0,458 ; 0,459)$ \\
\hline $2\left[{\left.\operatorname{máx~} \mathrm{g}^{*}(\mathrm{x})\right]}^{3,27}\right.$ & 40 & 0 & 756 & 1 & 0,238 & $(0,234 ; 0,243)$ & 0,469 & $(0,468 ; 0,469)$ \\
\hline $3\left[{\left.\operatorname{min~} \mathrm{h}^{*}(\mathrm{x})\right]}^{9,61}\right.$ & 10 & 0 & 167 & 1 & $-0,112$ & $(-0,119 ;-0,105)$ & 0,177 & $(0,168 ; 0,186)$ \\
\hline
\end{tabular}

* valores normalizados.

Los valores de la Tabla 1 muestran que en todas las configuraciones la variable $x_{3}$ fue igual a cero, por lo cual agregar un horno adicional no mejora el desempeño del sistema, ajustándose las demás variables a los valores obtenidos. En cambio las configuraciones obtenidas sí indican incorporar un torno y un operario adicional. Puede observarse que los indicadores de desempeño de cada escenario satisfacen la optimización buscada con sus respectivos modelos matemáticos. No obstante, el escenario 3 (óptimo ambiental) no es económicamente rentable, por lo que no se consideró en análisis posteriores, e indica únicamente una cota inferior de la generación de GEI del sistema de producción.

En las Tablas 2 y 3 se muestran los resultados de las comparaciones entre escenarios. En la Tabla 2 se comparan el beneficio y emisiones de $\mathrm{CO}_{2 \mathrm{eq}}$ de los escenarios 0,2 y 3 , 
contra la configuración multiobjetivo hallada (escenario 1). La Tabla 3 contiene los datos de la Tabla 2 y agrega la comparación de los demás escenarios, respecto a los mismos indicadores En estas Tablas puede observarse que el escenario multiobjetivo respecto al modelo inicial produjo mayor beneficio, pero también mayores emisiones de $\mathrm{CO}_{2 \mathrm{eq}}$, con diferencias significativas para ambos resultados. Sin embargo, también se destacan en estas Tablas que, salvo los beneficios de los escenarios 1 y 2 , las diferencias de desempeño tanto ambiental como económico son significativas entre los escenarios. Se deduce, como primer resultado importante, que con el modelo multiobjetivo se obtuvo una configuración que no exhibe diferencia significativa respecto al óptimo económico, y que además genera una reducción, pequeña pero significativa, de GEI. Esto permite responder afirmativamente la pregunta de investigación.

Las medias de la Tabla 1 indican que empleando el escenario 2 se obtuvieron incrementos en el beneficio semanal y en las emisiones de GEI, respecto al sistema inicial, de $51,59 \%$ y $37,94 \%$ respectivamente; mientras que con el escenario 1 los incrementos en promedio de los mismos indicadores fueron de $47,77 \%$ y $34,70 \%$ respectivamente. Al comparar los resultados de los escenarios 1 y 2 , se observa que el primero proporciona una solución de compromiso, ya que en promedio el escenario óptimo multiobjetivo genera menos beneficio semanal que el óptimo económico, a costa de generar menos emisiones de GEI.

Tabla 2. Comparaciones de escenarios, contra el óptimo multiobjetivo $\left(\mathrm{H}_{0}: \mu_{i j}=\mu_{1 j} ; ; \mathrm{H}_{1}: \mu_{i j} \neq\right.$ $\mu_{1 j} ; i=0,2,3 ; j=1$ (beneficio), $2\left(\right.$ emisiones de $\left.\mathrm{CO}_{2 \mathrm{eq}}\right) ; \alpha^{\prime}=0,016$ )

\begin{tabular}{|c|c|c|c|c|}
\hline & \multicolumn{2}{|c|}{ Beneficio $^{*}$} & \multicolumn{2}{|c|}{ Emisiones de $\mathrm{CO}_{2 \mathrm{eq}}{ }^{*}$} \\
\hline \multirow{2}{*}{ Escenarios (i) } & $\bar{X}_{i}-\bar{X}_{1}$ & Intervalos de diferencias & $\bar{X}_{i}-\bar{X}_{1}$ & Intervalos de diferencias \\
\hline 0 & $-0,074$ & $(-0,090 ;-0,058)^{\star *}$ & $-0,118$ & $(-0,123 ;-0,113)^{\star *}$ \\
\hline 2 & 0,007 & $(0,000 ; 0,015)$ & 0,010 & $(0,010 ; 0,011)^{\star *}$ \\
\hline 3 & $-0,343$ & $(-0,353 ;-0,333)^{\star *}$ & $-0,281$ & $(-0,293 ;-0,270)^{* *}$ \\
\hline
\end{tabular}

*valores normalizados; ${ }^{* *}$ indica diferencia significativa.

Tabla 3. Comparaciones entre pares de escenarios $\left(\bar{X}_{i 2}-\bar{X}_{i 1}, \mathrm{i}_{1}<\mathrm{i}_{2} ; \mathrm{H}_{0}: \mu_{i 2 j}=\mu_{i 1 j} ; \mathrm{H}_{1}: \mu_{i 2 j} \neq \mu_{i 1 j}\right.$ $\left.i=0,1,2,3 ; j=1,2 ; \alpha^{\prime}=0,008\right)$

\begin{tabular}{|c|c|c|c|}
\hline & \multicolumn{3}{|c|}{ Beneficio*. Intervalos de diferencias } \\
\hline & \multicolumn{3}{|c|}{$\mathrm{i}_{2}$} \\
\hline Escenarios $\left(\mathrm{i}_{1}\right)$ & 1 & 2 & 3 \\
\hline 0 & $(0,055 ; 0,092)^{\star *}$ & $(0,062 ; 0,100)^{* *}$ & $(-0,290 ;-0,249)^{\star \star}$ \\
\hline 1 & & $(-0,001 ; 0,016)$ & $(-0,355 ;-0,332)^{* *}$ \\
\hline \multirow[t]{3}{*}{2} & & & $(-0,364 ;-0,337)^{\star \star}$ \\
\hline & \multicolumn{3}{|c|}{ Emisiones de $\mathrm{CO}_{2 \mathrm{eq}}{ }^{*}$. Intervalos de diferencias } \\
\hline & \multicolumn{3}{|c|}{$\mathrm{i}_{2}$} \\
\hline Escenarios $\left(\mathrm{i}_{1}\right)$ & 1 & 2 & 3 \\
\hline 0 & $(0,112 ; 0,123)^{\star *}$ & $(0,123 ; 0,134)^{* *}$ & $(-0,178 ;-0,149)^{\star *}$ \\
\hline 1 & & $(0,009 ; 0,011)^{* *}$ & $(-0,295 ;-0,268)^{\star *}$ \\
\hline 2 & & & $(-0,305 ;-0,278)^{\star *}$ \\
\hline
\end{tabular}


Los resultados condicen, en términos generales, con experiencias reportadas en la literatura. Aplicaciones de simulación en diferentes tipos de sistemas de manufactura mejoran el consumo de energía, como Herrmann et al. (2011) y Solding (2008), y reducen emisiones de $\mathrm{CO}_{2 \mathrm{eq}}$, como Sproedt et al. (2015). En estos casos, en simultáneo incrementan o mantienen el nivel de producción o la eficiencia de los sistemas que modelan, lo cual redundaría en mayor o igual beneficio económico. Sin embargo, al no considerar los costos que se incurrirían en estas mejoras es difícil inferir en forma directa respecto al beneficio. Los resultados del presente trabajo se destacan en tal sentido.

Para ilustrar lo anterior, considérese la simulación de la fábrica de tejidos de Herrmann et al. (2011). En los escenarios que proponen varían la velocidad del proceso, recurriendo también a sistemas alternativos de generación y recuperación de vapor y aire comprimido. La solución que reportan mantiene el nivel de producción, pero reduce los costos de energía. Sin embargo, no informan sobre los otros costos que implicarían estas modificaciones en el proceso. Cabe mencionar también que en la optimización de los trabajos señalados las variables que utilizan son diversas, entre modificaciones tecnológicas, sobre parámetros del proceso y en planificación de la producción.

Otras producciones de la literatura usan métricas económicas y ambientales, empero un único objetivo en la optimización. Por ejemplo, Díaz et al. (2013), combinan estrategias típicas de lean manufacturing junto con otras que denominan green, cuyo fin es reducir el consumo energético. No obstante, en la optimización se enfocan la reducción de costos, lo cual logran en 10,8\%. También Wróbel y Oleśków (2014), evalúan métricas de productividad, atendiendo estándares ambientales, también de salud y seguridad. Asimismo, obtienen diseños del proceso con los que reducen costos.

Respecto a la optimización considerando únicamente la emisión de GEl, los resultados del escenario 3 muestran una reducción de casi $48 \%$ respecto al escenario 0 y de alrededor de $61 \%$ respecto al escenario 1, pero ya se mencionó que es económicamente inviable. Este resultado deja la vista el conflicto entre objetivos económicos y ambientales en el sistema de manufactura, el cual debe estudiarse cuidadosamente si se quiere reducir las emisiones de GEI sin sacrificar la rentabilidad. Entre las publicaciones sobre simulación en sistemas de manufactura, Sproedt et al. (2015) muestran casos donde reducen las emisiones de GEI entre $2 \%$ y $3 \%$; y Solding (2008) presenta un caso donde reduce el uso de energía (lo que se puede traducir en emisiones de $\mathrm{CO}_{2 \text { eq }}$, como se realizó en este trabajo) de $5 \%$, manteniendo la productividad de la planta. Estos resultados condicen con lo obtenido en el caso estudiado.

Entre las publicaciones más relevantes a los fines comparativos sobre simulación en plantas de procesamiento de madera, Baesler et al.(2002) modelan un proceso de fabricación de artículos de madera, obteniendo una mejora en la productividad del proceso del $18 \%$ respecto de las condiciones iniciales. Thoews et al.(2008) reportan un incremento en la producción de hasta $37 \%$, a partir de modificaciones en parámetros de funcionamiento del proceso, utilizando algoritmos metaheurísticos. Si se consintiese en que esta mejora conduciría a un incremento proporcional en el beneficio, entonces dicho valor se asemeja más al aumento de $\mathrm{f}$ del escenario 1 respecto a las condiciones iniciales (Tabla 1), que en promedio es de 47,8\%.

Como no se conocen antecedentes de este tipo de aplicaciones de simulación en plantas de procesamiento de madera, entonces, los resultados que se presentan pueden servir para comparaciones con trabajos que se realicen a futuro. 


\section{CONCLUSIONES}

En este trabajo se presenta la simulación y optimización del sistema de producción de una pequeña empresa maderera. Tiene la particularidad de que se emplean en simultáneo criterios económico y ambiental. Se trata de un enfoque relativamente reciente en simulación de sistemas de manufactura y no se conocen antecedentes en industrias de procesamiento de madera.

Mediante la implementación del modelo desarrollado, se demuestra como los entornos de simulación por eventos discretos actuales, son suficientemente flexibles para integrar los cálculos asociados a indicadores ambientales, además de los ya usuales indicadores económicos. Por otra parte, coincidentemente con otras investigaciones existentes, se pone a la vista como los algoritmos metaheurísticos de optimización son capaces de operar bajo los niveles de incertidumbre de los modelos de simulación, posibilitando encontrar configuraciones con sustanciales mejoras en el desempeño de sistemas de manufactura.

En el caso de estudio analizado, la configuración que se obtiene con el modelo multiobjetivo alcanza un rendimiento económico mayor en casi $48 \%$ respecto a la configuración inicial. En relación al criterio ambiental, la optimización multiobjetivo permite obtener una configuración que, si bien genera más emisiones que la situación inicial, el incremento es aproximadamente $3 \%$ menor que el nivel de emisiones que proporciona el escenario óptimo económico.

El enfoque adoptado demuestra que permite obtener mejoras tanto en indicadores económicos como ambientales en simultáneo en los sistemas de producción del sector maderero, incluso en casos de pequeños establecimientos. Los resultados que se presentan conforman una base de comparación para futuros trabajos.

Entre las líneas de investigación futuras pueden mencionarse la simulación y optimización de sistemas de manufactura con los mismos y/u otros criterios, incluyendo distintos métodos para modelizar las preferencias de los decisores; la aplicación y comparación de otros tipos de funciones objetivo; la optimización multiobjetivo con restricciones estocásticas; y la aplicación y comparación de otros métodos de optimización.

\section{AGRADECIMIENTOS}

A los Ingenieros Ronny Boris Ben Bochert, Mauricio Maikol Guroski y Hugo Oscar Batista, por su labor en el caso estudiado. También al Doctor Rafael Alejandro Vargas, por sus sugerencias para la elaboración de este trabajo.

\section{REFERENCIAS}

AMARAN, S., SAHINIDIS, N.V., SHARDA, B. y BURY, S. J. (2016). Simulation optimization: a review of algorithms and applications. Annals of Operations Research, v. 240, n.1, pp.351380.

ANDERSON, R. B. Furniture rough mill costs evaluated by computer simulation. USDA Forest Service Research Paper NE- 518, 1983, 1-11.

ANDRADÓTTIR, S. A review of random search methods. En Handbook of simulation optimization. Springer: New York, 2015. pp. 277-292. ISBN 978-1-4939-1384-8. 
AUNE, J. E. (1974). System simulation - a technique for sawmill productivity analyses and designs. The Forestry Chronicle, v.50, n.2, pp. 66-69.

BAESLER, F. F. y SEPÚLVEDA, J. A. Multi-response simulation optimization using stochastic genetic search within a goal programming framework. En Proceedings of the 2000 Winter Simulation Conference. IEEE, 2000. pp. 788-794.

BAESLER, F. F., MORAGA, M. y RAMIS, F. J. Productivity improvement in the wood industry using simulation and artificial intelligence. En Proceedings of the 2002 Winter Simulation Conference. IEEE, 2002. pp. 1095-1098.

BANKS, J., CARSON, J. S., NELSON, B. L. y NICOL, D. M. Discrete-event system simulation. $4^{\text {th }}$ ed. Pearson. 2005. ISBN 978013446793.

BETTER, M., GLOVER, F. y KOCHENBERGER, G. Simulation optimization: improving decisions under uncertainty. En Breakthroughs in decision science and risk analysis. John Wiley \& Sons: New Jersey, 2015. p. 59-81. ISBN 978-1-118-21716.

CHICA, M., JUAN, A. A., CORDÓN, Ó. y KELTON, W. D. Why simheuristics? Benefits, limitations, and best practices when combining metaheuristics with simulation. SSRN[en línea]. 1 de enero de 2017 [Consulta: 8 de febrero de 2018]. Disponible en: Chica, http:// dx.doi.org/10.2139/ssrn.2919208.

CHOI, B. K. y KANG, D. Modeling and simulation of discrete-event systems. John Wiley \& Sons: New Jersey, 2013. ISBN 978-1-118-38699-6.

CHWIF, L., BANKS, J., DE MOURA FILHO, J. P. y SANTINI, B. (2013). A framework for specifying a discrete-event simulation conceptual model. Journal of Simulation, v.7, n.1, 5060.

DEB, K. Multi-objective Optimization. In: E. K. BURKE and G. Kendall, eds. Search methodologies. $2^{\text {nd }}$ ed. Springer: Boston, 2014. p. 403-449. ISBN 978-1-4614-6939-1.

DIAZ-ELSAYED, N., JONDRAL, A., GREINACHER, S., DORNFELD, D. y LANZA, G. (2013). Assessment of lean and green strategies by simulation of manufacturing systems in discrete production environments. CIRP Annals - Manufacturing Technology, v.62, pp. 475-478.

ELARBI, M., BECHIKH, S., SAID, L. B. y DATTA, R. Multi-objective optimization: classical and evolutionary approaches. En Recent advances in evolutionary multi-objective optimization. Springer: Switzerland, 2017. p. 1-30. ISBN 978-3-319-42977-9.

ELDER, M. DES view on simulation modeling: SIMUL8. En Discrete-event simulation and system dynamics for management decision making. John Wiley \& Sons: United Kingdom, 2014. pp. 199-214. ISBN 978-1-118-34902-1.

ELIZANDRO, D. y TAHA, H. Performance evaluation of industrial systems: discrete event simulation in using Excel/VBA. $2^{\text {nd }}$ ed. CRC Press: Boca Raton, 2012. ISBN 978-1-43987135-5.

ESMAEILIAN, B., BEHDAD, S. y WANG, B. (2016). The evolution and future of manufacturing: a review. Journal of Manufacturing Systems, v.39, n.1, pp.79-100.

FIGUEIRA, G. y ALMADA-LOBO, B. (2014). Hybrid simulation-optimization methods: a taxonomy and discussion. Simulation modelling practice and theory, v.46, pp.118-134.

FONTES, D. B. M. M. y GASPAR-CUNHA, . On multi-objective evolutionary algorithms. En Handbook of multicriteria analysis. Springer: Heidelberg, 2010.p. 287- 310. ISBN 978-3-54092827-0.

FRANCHETTI, M. J. y APUL, D. Carbon footprint analysis: concepts, methods, implementation, and case studies. CRC Press: Boca Raton, 2013. ISBN 978-1-4398-5784-7.

FU, M. C. (2002). Optimization for simulation: theory vs. practice. INFORMS Journal on 
Computing, v.14, n.3, pp.192-215.

FU, M. C. Handbook of simulation optimization. Springer: New York, 2015. ISBN 978-1-49391383-1.

FU, M. C., GLOVER, F. W. y APRIL, J. Simulation optimization: a review, new developments, and applications. En Proceedings of the 2005 Winter Simulation Conference. IEEE, 2005. pp. $1-14$.

FU, M. C., BAYRAKSAN, G., HENDERSON, S. G., NELSON, B. L., POWELL, W. B., RYZHOV, I. O. y THENGVALL, B. Simulation optimization: a panel on the state of the art in research and practice. En Proceedings of the 2014 Winter Simulation Conference. IEEE, 2014. p. 36963706.

GARCÍA, S., LUENGO, J. y HERRERA, F. Data preprocessing in data mining. Springer: Switzerland, 2015. ISBN 978-3-319-10246-7.

GARWOOD, T. L., HUGHES, B. R., OATES, M. R., O'CONNOR, D. y HUGHES, R. (2018). A review of energy simulation tools for the manufacturing sector. Renewable and Sustainable Energy Reviews, v. 81, pp. 895-911.

GUASCH, A., PIERA, M. À., CASANOVAS, J. y FIGUERAS, J. Modelado y simulación: aplicación a procesos logísticos de fabricación y servicios. $2^{\mathrm{a}}$ ed. Edición UPC: Barcelona, 2003. ISBN 84-8301-704-0.

HEILALA, J., VATANEN, S., TONTERI, H., MONTONEN, J., LIND, S., JOHANSSON, B. y STAHRE, J. Simulation-based sustainable manufacturing system design. En Proceedings of the 2008 Winter Simulation Conference. IEEE, 2008, pp. 1922-1930.

HERRMANN, C., THIEDE, S., KARA, S. y HESSELBACH, J. (2011). Energy oriented simulation of manufacturing systems-concept and application. CIRP Annals-Manufacturing Technology, v.60, n.1, pp. 45-48.

HILLIER, F. S., y LIEBERMAN, G. J. Introduction to operations research. $10^{\text {th }}$ ed. McGraw-Hill Education: New York, 2015. ISBN 978-0-07-352345-3.

HONG, L. J., NELSON, B. L. y XU, J. Discrete optimization via simulation. En Handbook of simulation optimization. Springer: New York, 2015. pp. 9-43. ISBN 978-1-4939-1383-1.

HUNTER, S. R., APPLEGATE, E. A., ARORA, V., CHONG, B., COOPER, K., RINCÓNGUEVARA, O. y VIVAS-VALENCIA, C. (2017). An introduction to multi-objective simulation optimization. Optimization online [en línea], [Consulta: 8 de febrero de 2018]. Disponible en: https://goo.gl/NXDsrx.

JOHANSSON, M. T. y THOLLANDER, P. (2018). A review of barriers to and driving forces for improved energy efficiency in swedish industry-recommendations for successful in-house energy management. Renewable and Sustainable Energy Reviews, v.82, pp. 618-628.

KIBIRA, D. y SHAO, G. Integrating data mining and simulation optimization for decision making in manufacturing. En Applied simulation and optimization 2: new applications in logistics, industrial and aeronautical practice. Springer: Switzerland, 2017. pp. 81-105. ISBN 978-3-319-55809-7

KLEIJNEN, J. P. C. Design and analysis of simulation experiments. $2^{\text {nd }}$ ed. Springer: Switzerland, 2015. ISBN 978-3-319-18086-1.

KLINE, D. E., WIEDENBECK, J. K. y ARAMAN, P. A. (1992). Management of wood products manufacturing using simulation/animation. Forest Products Journal, v.42, n.2, pp. 45-52.

LAGUNA, M. OptQuest: optimization of complex systems [en línea]. White paper, OptTek Systems Inc, 2011 [Consulta: 8 de febrero de 2018]. Disponible en: https://goo.gl/2rStef.

LAGUNA, M. y MARKLUND, J. Business process modeling, simulation and design. $2^{\text {nd }}$ ed. 
CRC Press: Boca Raton, 2013. ISBN 978-1-4398-8528-4.

LAROSE, D. T. y LAROSE, C. D. Data mining and predictive analytics. $2^{\text {nd }}$ ed. John Wiley \& Sons: New Jersey, 2015. ISBN 978-1-118-11619-7.

LAW, A. M. How to build valid and credible simulation models. En Proceedings of the 2008 Winter Simulation Conference. IEEE, 2008. pp. 39-47.

LAW, A. M. A tutorial on design of experiments for simulation modeling. En Proceedings of the 2014 Winter Simulation Conference. IEEE, 2014. pp. 66-80.

LAW, A. M. Simulation modeling and analysis. $5^{\text {th }}$ ed. McGraw-Hill Education: New York, 2015a. ISBN 978-0-07-340132-4.

LAW, A. M. Statistical analysis of simulation output data: the practical state of the art. En: Proceedings of the 2015 Winter Simulation Conference. IEEE, 2015b, pp. 1810-1819.

LIN, R., SIR, M. Y. y PASUPATHY, K. S. (2013). Multi-objective simulation optimization using data envelopment analysis and genetic algorithm: specific application to determining optimal resource levels in surgical services. Omega, v.41, n.5, pp. 881-892.

Ministerio de Energía y Minería de Argentina. Cálculo del factor de emisión de CO2 de la Red Argentina de Energía Eléctrica (C2016 [Consulta: 8 de febrero de 2018]. Disponible en: https://goo.gl/Hqq5jZ.

MOHAMAD, I. B. y USMAN, D. (2013). Standardization and its effects on k-means clustering algorithm. Research Journal of Applied Sciences, Engineering and Technology, v.6, n.17, p. 3299-3303.

MOURTZIS, D., DOUKAS, M. y BERNIDAKI, D. (2014). Simulation in manufacturing: review and challenges. Procedia CIRP, v.25, pp. 213-229.

NEGAHBAN, A. y SMITH, J. S. (2014). Simulation for manufacturing system design and operation: literature review and analysis. Journal of Manufacturing Systems, v.33, n.2, pp. 241-261.

NELSON, B.L. Foundations and methods of stochastic simulation: a first course. Springer Science \& Business Media: New York, 2013. ISBN 978-1-4614-6159-3.

OMOGBAI, O. y SALONITIS, K. (2016). Manufacturing system lean improvement design using discrete event simulation. Procedia CIRP, v.57, pp.195-200.

OptTek. OptQuest: the world's leading simulation optimization engine @2018 [en línea] [Consulta: 8 de febrero de 2018]. Disponible en: https://goo.gl/HrWjYv.

PATEL, K. M. A. y THAKRAL, P. The best clustering algorithms in data mining. En International Conference on Communication and Signal Processing. IEEE, 2016. p. 2042-2046.

PAWLEWSKI, P. y BORUCKI, J. "Green” possibilities of simulation software for production and logistics - a survey. En Information Technologies in environmental engineering: new trends and challenges. Springer: Berlin, Heidelberg, 2011. p. 675- 688. ISBN 978-3-642-19535-8.

REYNOLDS, H. W. y GATCHELL, C. J. Sawmill simulation: concepts and computer use. USDA Forest Service Research Note NE- 100, 1969, 1-5.

RÍOS INSÚA, D., RÍOS INSÚA, S., MARTIIN JIMÉNEZ, J. y JIMÉNEZ MARTÍN, A. Simulación. Métodos y aplicaciones. $2^{a}$ ed. Alfaomega: México, 2009. ISBN 978-970-15-1457-3.

SARGENT, R. G. An introductory tutorial on verification and validation of simulation models. En Proceedings of the 2015 Winter Simulation Conference. IEEE, pp. 1729-1740. (2015)

SIMUL8 Corporation. SIMUL8 simulation software (C2018 [en línea] [Consulta: 8 de febrero de 2018]. Disponible en: https://goo.gl/xU5FDB.

SOLDING, P. Increased energy efficiency in manufacturing systems using discrete event 
simulation: applied studies on the Swedish foundry industry [en línea]. Doctoral thesis. Leicester: De Montfort University, 2008. [Consulta: 8 de febrero de 2018]. Disponible en: https://goo.gl/6i9kLh.

SPROEDT, A., PLEHN, J., SCHÖNSLEBEN, P. y HERRMANN, C. (2015). A simulation-based decision support for eco-efficiency improvements in production systems. Journal of Cleaner Production, v.105, pp. 389-405.

THIEDE, S., SEOW, Y., ANDERSSON, J. y JOHANSSON, B. (2013). Environmental aspects in manufacturing system modelling and simulation-State of the art and research perspectives. CIRP Journal of Manufacturing Science and Technology, v.6, pp. 78-87.

THOEWS, S. E., MANESS, T. C. y RISTEA, C. (2008). Using flow simulation as a decision tool for improvements in sawmill productivity. Maderas. Ciencia y tecnología, v.10, n.3, pp. 229-242.

TSAI, S., XUE, Y., CHEN, Q. C. y ZHOU, J. Discussing and evaluating the green environmental performance of manufacturers. En Research advances in industrial engineering. Springer: Switzerland, 2015. p. 59-75. ISBN 978-3-319-17824-0.

UNZALU, P. Guía Metodológica para la aplicación de la norma UNE-ISO 14064-1:2006 para el desarrollo de Inventarios de Gases de Efecto Invernadero. Ihobe S. A: Bilbao, 2012.

VAN DIJK, N. M., HAIJEMA, R., VAN DER SLUIS, E., KORTBEEK, N., AL-IBRAHIM, A. y VAN DER WAL, J. OR and simulation in combination for optimization. En Applied simulation and optimization: in logistics, industrial and aeronautical practice. Springer: Switzerland, 2015. p.75-107. ISBN 978-3-319-15032-1.

WERY, J., MARIER, P., GAUDREAULT, J., CHABOT, C. y THOMAS, A. Improving a hardwood flooring cutting system through simulation and optimization. En Proceedings of the 2015 Winter Simulation Conference IEEE, pp. 2172-2182. (2015)

WRÓBEL, G. y OLEŚKÓW-SZŁAPKA, J. (2014). Simulation method for the benefits of a small business in sustainable world. En Process simulation and optimization in sustainable logistics and manufacturing. Springer: Switzerland, 2014. p. 3-21. ISBN 978-3-319-07346-0.

YEGUL, M. F., ERENAY, F. S., STRIEPE, S. y YAVUZ, M. (2017) Improving configuration of complex production lines via simulation-based optimization. Computers \& Industrial Engineering, v.109, pp. 295-312.

\section{BIOGRAFÍA}

Héctor Darío Enriquez. Docente investigador de la Facultad de Ingeniería de la Universidad Nacional de Misiones, Argentina. Ingeniero Industrial por la misma Institución y Magister en Logística Integral por la Universidad Autónoma de Barcelona, España. Ha participado y publicado en diferentes eventos académicos y revistas nacionales e internacionales.

Sebastián Federico Kolodziej. Docente investigador en la Facultad de Ingeniería de la Universidad Nacional de Misiones, Argentina. Ingeniero Industrial, Especialista en Higiene y Seguridad en el Trabajo y maestrando en Gestión Ambiental por la misma Institución. Ha participado y publicado en diferentes eventos académicos nacionales e internacionales. 